\title{
Slopes Analyses - Case Study, Slope Stability of Bypass Project
}

\section{Diana Bardhi}

PhD. Cand. Eng. Department of Civil Engineering, Metropolitan University of

Tirana, Albania

\section{Abstract}

The scope of this study was to compare various stability evaluation methods. Accordingly, most common LE approaches were compared with the advanced LE (M-P) method. Similarly, the differences in FOS computed from LE and FE analyses were compared based on a simple slope considering various load cases. In addition, two real slopes in a case study were analysed for the recorded minimum-maximum GWT, pseudo-static and dynamic conditions. Moreover, the stability evaluations of these slopes were based on both LE (MP) and FE (PLAXIS) calculation approaches, which both utilized shear strength parameters from advanced triaxle tests. Similarly, Mohr-Coulomb model was applied in both approaches. The following conclusions are hence derived based on the reported work on both idealized and real slopes. To fulfil one of the aims of the study, the LE based methods are compared based on the factor of safety (FOS) obtained for various load combinations. The comparison is mainly based on simplified slope geometry and assumed input parameters. Among the LE methods, the Bishop simplified (BS), Janbu simplified (JS) and Janbu GPS methods are compared with the Morgenstern-Price method (MPM). These LE methods are well established for many years, and thus some of them are still commonly used in practice for stability analysis. Moreover, the M-PM has been compared with results from the FE analyses. Compared with theFE (PLAXIS) analyses, the LE (M-PM) analyses may estimate $5-14 \%$ higher FOS, depending on the conditions of a dry slope and a fully saturated slope with hydrostatic pore pressure distributions. For fully saturated conditions in the slope, inaccurate computation of stresses in LE methods may have resulted in larger difference in the computed FOS. Since, the FE software is based on stress-strain relationship, stress redistributions are surely better computed even for a complicated problem. This has been found one of the advantages in FE simulations. A parameter study shows that the application of a positive dilatancy angle in FE analysis can significantly improve the FOS (4 - 10\%). On contrast, the shear surface optimization in LE (M-PM in SLOPE/W) analysis results in lower FOS, and thus minimizing the difference in FOS compared with FE analysis

Keywords: slope, stability, techniques, finite element analyses, stress 


\section{Introduction}

Instability related issues in engineered as well as natural slopes are common challenges to both researchers and professionals. In construction areas, instability may result due to rainfall, increase in groundwater table and change in stress conditions. Similarly, natural slopes that have been stable for many years may suddenly fail due to changes in geometry, external forces and loss of shear strength. The combination of intense rainfalls, steep topography and soil conditions are critical. Albania has been facing challenges of large number of water-induced disasters such as landslides or slope failures mainly along the Highways. Likewise Earthquakes are the greatest threat to the long-term stability of slopes in earthquake active zones. In addition, the long-term stability is also associated with the weathering and chemical influences that may decrease the shear strength and create tension cracks. In such circumstances, the evaluation of slope stability conditions becomes a primary concern everywhere.

The engineering solutions to slope instability problems require good understanding of analytical methods, investigative tools and stabilization measures. A quantitative assessment of the safety factor is important when decisions are made. The primary aim of slope stability analyses is to contribute to the safe and economic design of excavation, embankment and earth dams.

Development activities may face great challenges due to unstable grounds. Similarly, the slope failure may interrupt the established imperative services like traffic movement, drinking water supply, power production and similar infrastructures. In this way, the main motivation of stability analyses is to save human lives, reduce property damages and provide continuous services. Therefore, the most suitable and reliable stability analysis methods have great scope and thus, they are increasingly demanding. The chosen method should be able to identify the existing safety conditions and suggest for technically feasible and economically viable solutions.

\section{The scope and the aim of this paper}

The scope of this paper: "Slopes Analyses using other Techniques" by using evaluations of limit equilibrium (LE) and advanced finite element (FE) methods" is expected to address such instability problems. The analytical solutions to the instability problems, including the effects of groundwater variations and earthquake effects, are expected to contribute to improve the knowledge of these processes, with respect to the benefit of engineering in Albania, Vlora Bypass project.

\section{There are two main objectives to be fulfilled from this research paper}

The first: to compare the most common limit equilibrium (LE) methods with the advanced finite element (FE) method, and a short brief presentation of threedimensional slope stability analysis by elasto-plastic finite elements. 
The second: to evaluate the stability conditions of the slopes situated at the Vlora Bypass Project, as a case study.

Within this framework, the paper has focused on the following topics:

Comparison and evaluation of slope stability (LE and FE) methods,

Evaluation to the long-term stability condition of natural slopes, and

Investigations of shear strength and other relevant parameters for stability analyses.

Available approaches are utilized to achieve the outlined objectives in this study. The first two objectives are fulfilled using following three computer based geotechnical software codes:

SLOPE/W, based on LE principles,

SLIDE, also based on LE principles, and

PLAXIS, based on FE principles.

Among the most common LE based methods, which are incorporated in both SLOPE/W and SLIDE software, Bishop and Janbu's simplified methods, Janbu's generalized method and the Morgenstern-Price method are considered for comparison purposes. The factor of safety (FOS) from these methods is further compared with the FOS obtained by the FEM code PLAXIS. The comparisons are based on a simplified slope analysed for various load combinations.

Moreover, the long term-stability of natural slopes has been evaluated under the second objective with the most adverse load combinations. The stability evaluations of real slopes are again based on the selected LE methods from the simplified slope analyses. The third objective is achieved from the field and laboratory investigations. Several tests were conducted to determine the relevant input parameters.

\section{Limit equilibrium method}

Several limit equilibrium (LE) methods have been developed for slope stability analyses. Fellenius (1936) introduced the first method, referred to as the Ordinary or the Swedish method, for a circular slip surface. Bishop (1955) advanced the first method introducing a new relationship for the base normal force.

The equation for the FOS hence became non-linear. At the same time, Janbu (1954a) developed a simplified method for non-circular failure surfaces, dividing a potential sliding mass into several vertical slices. The generalized procedure of slices (GPS) was developed at the same time as a further development of the simplified method (Janbu 1973). Later, Morgenstern-Price (1965), Spencer (1967), Sarma (1973) and several others made further contributions with different assumptions for the interslice forces. 
A procedure of General limit equilibrium (GLE) was developed by Chugh (1986) as an extension of the Spencer and Morgenstern-Price methods, satisfying both moment and force equilibrium conditions (Krahn 2004, Abramson et al. 2002). These developments are reviewed in the following section, which aims to find out the key differences in the various approaches for FOS determination.

All LE methods are based on certain assumptions for the interslice normal (E) and shear ( $\mathrm{T}$ ) forces, and the basic difference among the methods is how these forces are determined or assumed. In addition to this, the shape of the assumed slip surface and the equilibrium conditions for calculation of the FOS are among the others.

A summary of selected LE methods and their assumptions are presented in Table 1.2

Table 1.2 Summary of LE methods (Abramson et al. 2002, Nash 1987)

\begin{tabular}{l|cccc|l}
\hline Methods & Circular & Non-cir. & $\Sigma \mathrm{M}=0$ & $\Sigma \mathrm{F}=0$ & Assumptions for T and E \\
\hline Ordinary & $\sqrt{ }$ & - & $\sqrt{ }$ & - & Neglects both E and T \\
Bishop simplified & $\sqrt{ }$ & $\left(^{*}\right)$ & $\sqrt{ }$ & $\left(^{* *}\right)$ & Considers E, but neglects T \\
Janbu simplified & $\left(^{*}\right)$ & $\sqrt{ }$ & - & $\sqrt{ }$ & Considers E, but neglects T \\
Janbu GPS & $\sqrt{ }$ & $\sqrt{ }$ & $\left(^{* *}\right)$ & $\sqrt{ }$ & Considers both E and T, act at LoT \\
Lowe-Karafiath & - & $\sqrt{ }$ & - & $\sqrt{ }$ & Resultant inclines at, $\theta=1 / 2(\alpha+\beta)$ \\
Corps of Engrs. & - & $\sqrt{ }$ & - & $\sqrt{ }$ & Resultant inclines at, $\theta=1 / 2(\alpha 1+\alpha 2)$ \\
Sarma & $\sqrt{ }$ & $\sqrt{ }$ & $\sqrt{ }$ & $\sqrt{ }$ & Interslice shear, $T=c h+E \tan \phi$ \\
Spencer & $\sqrt{ }$ & $\left(^{*}\right)$ & $\sqrt{ }$ & $\sqrt{ }$ & Constant inclination, $\mathrm{T}=\tan \theta \mathrm{E}$ \\
Morgenst.-Price & $\sqrt{ }$ & $\sqrt{ }$ & $\sqrt{ }$ & $\sqrt{ }$ & Defined by $\mathrm{f}(\mathrm{x}), \mathrm{T}=\mathrm{f}(\mathrm{x}) \cdot \lambda . \mathrm{E}$ \\
\hline
\end{tabular}

$(*)$ Can be used for both circular and non-circular failure surfaces,

$(* *)$ satisfies vertical force equilibrium for base normal force, and

$(* * *)$ satisfies moment equilibrium for intermediate thin slices (Janbu 1957,

Grande 1997)

The inter-slice forces depend on a number of factors, including stress-strain and deformation characteristics of the materials. Their evaluation, however, becomes complicated in the LE methods. Therefore, simplified assumptions are made in most methods either to neglect both or to one of them. Nevertheless, the most advanced methods consider these forces in the LE analyses. Some of the basic principles behind the methods are briefly described below.

\section{General limit equilibrium procedure}

The general limit equilibrium (GLE) procedure incorporates all the assumptions and development made by the latest LE methods. In fact, this is an extension of Spencer and Morgenstern-Price methods where, the interslice slope, $\tan \theta=\lambda . \mathrm{f}(\mathrm{x})$ is assigned 
to determine the interslice forces (Krahn 2004, Abramson et al. 2002). In this way, the GLE procedure is good to compare the most common methods in a force equilibrium FOS versus $\lambda$ diagram as shown in Fig. 2.3.

The most likely inclinations of force equilibrium FOS $\left(F_{f}\right)$ and moment equilibrium FOS $\left(F_{m}\right)$ has been indicated particularly for circular shear surface (SS). (i.e. noncircular) SS analysis. The plane SS analysis may have revered position of $\mathrm{Ff}$ and $\mathrm{Fm}$ (Krahn 2004).

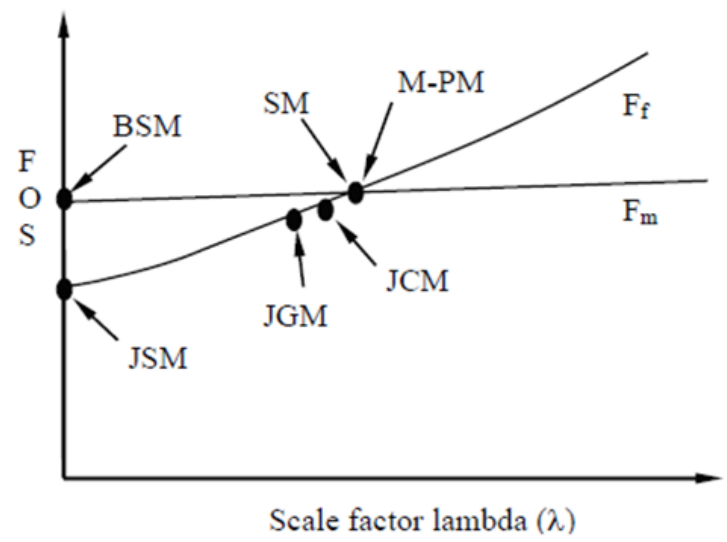

Fig.1.3 Presentation of the most common methods (Fredlund and Krahn 2004)

When $\lambda=0$, the FOS is obtained for Bishop's simplified method (BSM) and Janbu's simplified method (JSM)JSM, as indicated in Fig. 1.3. Similarly, the intersection point gives the FOS for SM or M-PM. According to Fredlund and Krahn (2004), Janbu's corrected and generalized methods are close to the intersecting point (see Fig. 1.3).

Thus, the GLE procedure has an advantage of making comparisons of FOS in the same diagram.

In summary, GLE procedure:

considers both interslice normal and shear forces,

satisfies both moment and force equilibriums,

allows selection for interslice force function, and

shows comparison of most common and advanced LE methods.

\section{Software used for stability analysis}

Slope stability analyses today can be performed by using various computer based geotechnical software. Today, both LE and FE based software are commonly used in geotechnical computations. 
SLOPE/W, developed by GEO-SLOPE International Canada, is used for slope stability analysis.This software is based on the theories and principles of the LE methods.

SLOPE/W has been applied separately and together with SEEP/W, other software program, which computes the pore pressure distributions, based on finite elements mesh and groundwater seepage analyses. Finally, the pore pressure distributions were coupled with slope stability analysis and FOS was determined. The software SLOPE/W computes FOS for various shear surfaces, for example circular, non-circular and user-defined surfaces (SLOPE/W 2002, Krahn 2004).

\section{Comparison of LE methods}

The LE methods in SLOPE/W can be compared in two ways. The first is based on the software searched for the circular (CSS) searched by each method and the corresponding FOS; the other is based on the software searched for the circular (CSS) searched by GLE procedure and the corresponding FOS. The first comparison can be done directly by looking into the "minimum FOS" for the selected methods. This appears immediately after completion of the computation. The second comparison can either be done by the $\lambda$ versus FOS plot or by the FOS found under the "slip surface with force data available" (optimized or non optimized FOS).

In fact, SLIDE is found similar to the SLOPE/W though there are few additional features, for example groundwater analysis and back analysis for support forces. Modelling in SLIDE for the study was possible for external loading, groundwater and forces, like surcharge and from pseudo-static earthquakes. The circular CSS was located automatically and the corresponding FOS was computed by the software in the similar way as in SLOPE/W.

The groundwater module was used to simulate and compute groundwater analysis based on finite element mesh, and the pore pressure distributions from the seepage analysis were used for the stability analysis. The analytical results are compared with SLOPE/W and PLAXIS.

PLAXIS is a finite element code for soil and rock analyses (PLAXIS 2004), developed by PLAXIS BV in cooperation with several universities including DUT in the Netherlands and NTNU in Norway. The computer program is applicable to many geotechnical problems, including stability analyses and steady-state groundwater flow calculations. This software contains several FE models and four main subroutines. These routines are inputs, calculations, outputs and curve plots. The FOS versus displacement is plotted from the curve plots sub-routine.

The slope models analysed were created by the input sub-routine. Material properties including shear strength parameters were defined for each soil layer. A plain strain model of 15 node triangular elements was used to generate the finite element mesh. Similarly, pore pressure distributions were generated based on phreatic level with and without corrections and the steady-state groundwater calculation. Moreover, a 
Mohr-Coulomb material model was selected for the stability analyses. The selected $\mathrm{M}-\mathrm{C}$ model is based on the elastic-perfectly plastic theory of soil mechanics.

Accordingly, both elastic parameters (E, $v$ ) and plastic parameters $\left(c^{\prime}, \varphi^{\prime}, \Psi\right)$ are utilized in the model. Similarly, in addition to the yield function $(f)$, the model has incorporated plastic potential function $(g)$, where the dilatancy angle $(\Psi)$ is associated with the plastic behaviour of soils. The formulation of the M-C model consists of six yield functions and six plastic functions.

One of each function is given below for demonstration purposes (PLAXIS 2004) only:

$$
\begin{aligned}
& f_{1}=\frac{1}{2}\left(\sigma_{2}^{\prime}-\sigma_{3}^{\prime}\right)+\frac{1}{2}\left(\sigma_{2}^{\prime}+\sigma_{3}^{\prime}\right) \sin \phi-c \cos \phi \leq 0 \\
& g_{1}=\frac{1}{2}\left(\sigma_{2}^{\prime}-\sigma_{3}^{\prime}\right)+\frac{1}{2}\left(\sigma_{2}^{\prime}+\sigma_{3}^{\prime}\right) \sin \psi
\end{aligned}
$$

\section{Computation of FOS}

FOS was computed by using the 'c- $\varphi$ reduction' procedure. According to PLAXIS (2004), this approach involves in successively reducing the soil strength parameters $c^{\prime}$ and $\tan \varphi$ ' until the failure occurs. The strength parameters are automatically reduced until the final calculation step results in a fully developed failure mechanism. Further, Nordal and Glaamen (2004) say, "By lowering the strength incrementally, a soil body is identified to fail after a certain strength reduction". In this way, PLAXIS computes the FOS as the ratio of the available shear strength to the strength at failure by summing up the incremental multiplier (Msf) as defined by:

$$
\text { FOS }=\frac{\text { available shear strength }}{\text { shear strength at failure }}=\text { value of } \sum M_{s f} \text { at failure }
$$

\subsection{Comparison of Analysis Methods}

\subsubsection{Geometry and input parameters}

Fig. 2.1 shows the idealized slope of $10 \mathrm{~m}$ height with an inclination of $1: 2$ (V:H). Two soil layers with different strength parameters are assumed. Furthermore, the same effective stress parameters are used in dry and wet conditions. Similarly, two unit weights of soils, one above the GWT $(\gamma d)$, the other below the GWT $(\gamma)$, are considered. Moreover, the same permeability coefficient $(\mathrm{k})$ is assumed in both layers for the seepage analysis. The parameters used in the study are shown in Fig. 2.1, with the complete set of input parameters given in Table 2.1. 


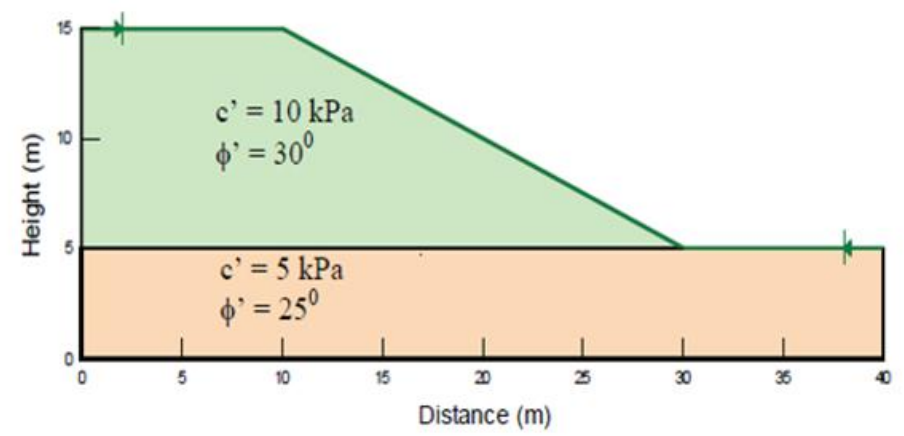

Fig.2.1 Idealised slope geometry for "model" with two soil layers

Table 2.1 Input parameters for analyses

\begin{tabular}{ccccccccc}
\hline Soil layers & $c^{\prime}(\mathrm{kPa})$ & $\phi^{\prime}\left({ }^{0}\right)$ & $\psi\left({ }^{9}\right)$ & $\gamma\left(\mathrm{kN} / \mathrm{m}^{3}\right)$ & $\gamma_{\mathrm{d}}\left(\mathrm{kN} / \mathrm{m}^{3}\right)$ & $\mathrm{E}_{\text {ref }}(\mathrm{kPa})$ & $v$ & $\mathrm{k}(\mathrm{m} / \mathrm{s})$ \\
\hline Upper & 10 & 30 & 0 & 20 & 18 & 5000 & 0.3 & $1 \times 10^{-5}$ \\
Lower & 5 & 25 & 0 & 20 & 18 & 5000 & 0.3 & $1 \times 10^{-5}$ \\
\hline
\end{tabular}

\subsubsection{Load combination for analysis}

Three different conditions; Dry slope, Wet slope and Dry slope with external loads are considered for the stability analyses. The load conditions analyzed are defined as:

Case 1: Completely dry slope, i.e. no GWT inside the model,

Case 2: Completely saturated slope, i.e. GWT on the surface (hydrostatic pore pressure),

\subsection{Selected methods for analysis, Application of software programs}

Stability chart methods, LE and FE based software are selected for analyses and comparison of the FOS. The basic theory and different assumptions made in the LE and FE methods. The selected methods and software are:

LE methods: Stability charts from JDM, two software SLOPE/W and SLIDE, and the selected methods are BSM, JSM, JGM and M-PM or GLE.

FE method: Software PLAXIS.

Load Case 1: Dry slope The stability of the dry slope was first analyzed in SLOPE/W. The minimum FOS and critical SS searched by entry and exit option are given in Fig. 3.1 


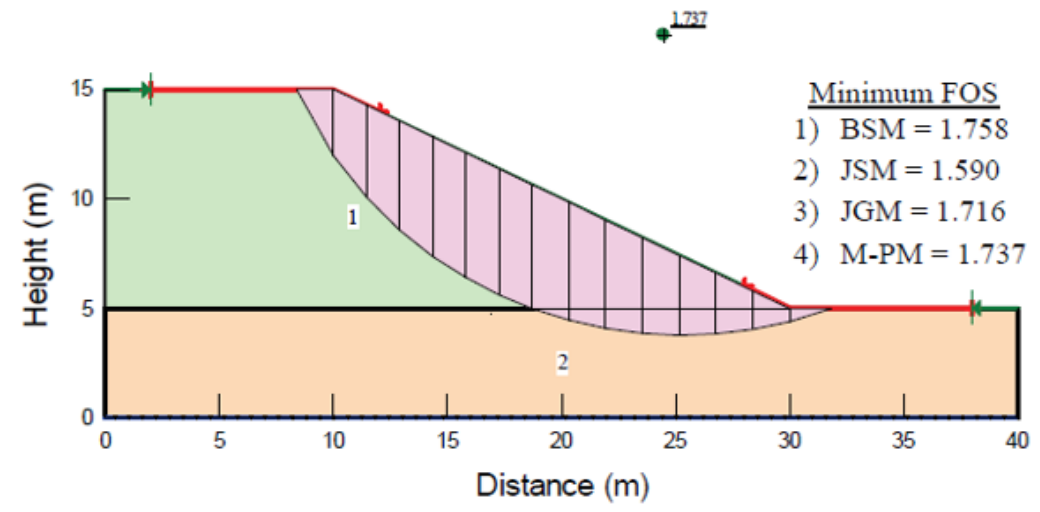

Fig.3.1 CSS by M-PM and FOS from SLOPE/W for Case 1

The CSS was searched from thousands of possible SS by defining the input of 15 slices, 1500 iterations, and 15 increments for entry, 10 increments for exit and 5 increments for radius. These parameters are consecutively chosen until the further increments do not change the FOS. A halfsine function was selected to compute the interslice forces with tolerance error of $1 \%$. Moreover, the selection of a half-sine function was based on the assumption that the interslice shear forces could be at maximum in the middle of the CSS and zero at the entry and exit points.

As in SLOPE/W, the analysis was carried out in SLIDE for the same input parameters and model geometry. The results from the analyses are presented in Fig. 3.2.

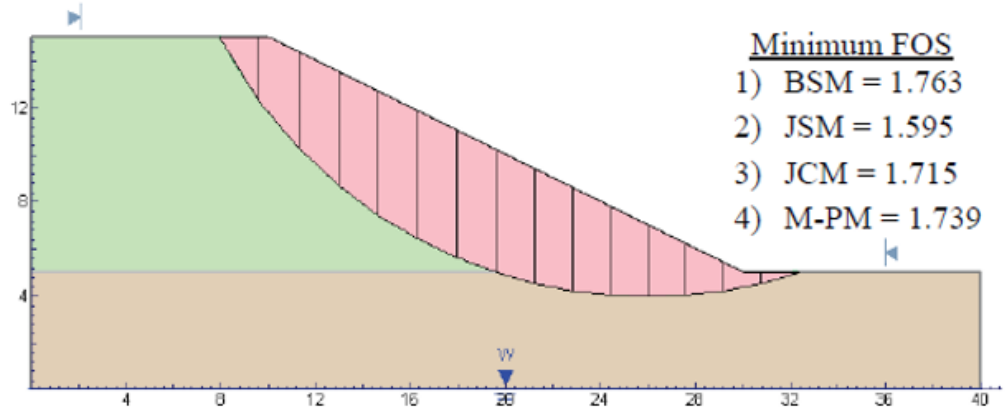

Fig.3.2 CSS by M-PM and FOS from SLIDE for Case 1

In SLIDE, the CSS was selected from the auto-refined search option by defining as similar inputs as before in SLOPE/W. The input parameters were increased until further increments had no change in the FOS. In this way, the CSS and the minimum FOS were obtained. The FOS from SLIDE was found very similar to SLOPE/W. In addition, the FOS from JCM in SLIDE and JGM in SLOPE/W was found to be the same. Moreover, the FOS from JGM was found only 1\% lower than that from M-PM for the CSS identified by each method. 
The analysis also included use of PLAXIS with the same Mohr-Coulomb soil model and strength parameters as given in Table 2.1. A plain strain model was defined by using 15 noded elements and a well-refined mesh of about 3000 elements with $450 \mathrm{~mm}$ average size, no tension cracks and 1\% tolerance. Further mesh refinement had no change in FOS. The FE based software PLAXIS computes FOS by the c- $\varphi$ reduction procedure. The CSS located by PLAXIS and the corresponding FOS are given in Fig. 3.3

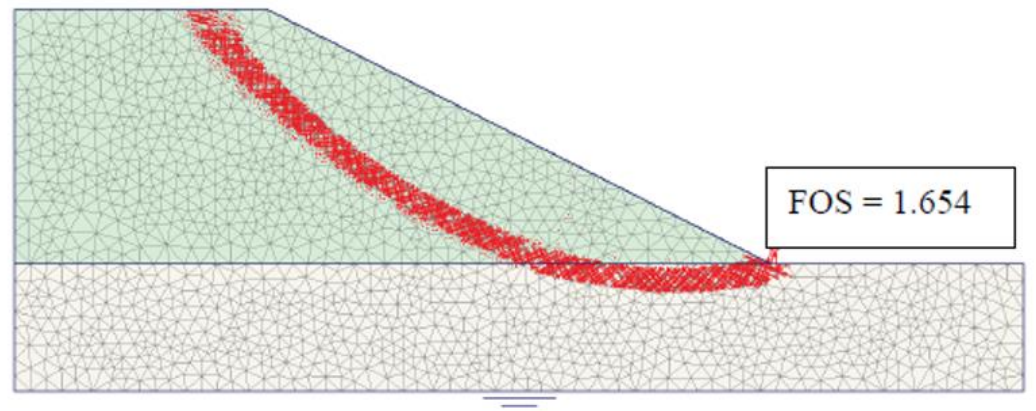

Fig. 3.3 CSS and FOS computed from PLAXIS for Case 1

PLAXIS identifies the CSS based on the strain localisation in the slope. The incremental strains are excessively concentrated inside the soil body (see Fig. 3.3) from which a failure may possibly initiate. Compared to the FOS obtained from PLAXIS, the LE method (M-PM) overestimates the FOS by $5 \%$ for dry slope conditions in this idealised slope analysis.

Load Case 2: Wet slope For Case 2b, the pore pressure profile obtained from the seepage analyses was used. Accordingly, the pore pressure distributions were first analysed separately in SEEP/W, and then the analysed pore pressure was used in SLOPE/W for compilation of the corresponding FOS. Taking the LE methods, the FOS was found $9 \%$ higher from seepage analyses than for hydrostatic distributions. SLIDE produced almost identical results, and they are hence not included here. The reason of higher FOS in Case 2b can be explained by the unchanged CSS even after the seepage analysis. This resulted in higher effective normal stress due to the lower average pore pressures along the CSS. The flow field and equipotential lines generated by the seepage analysis in SEEP/W Fig. 3.4 


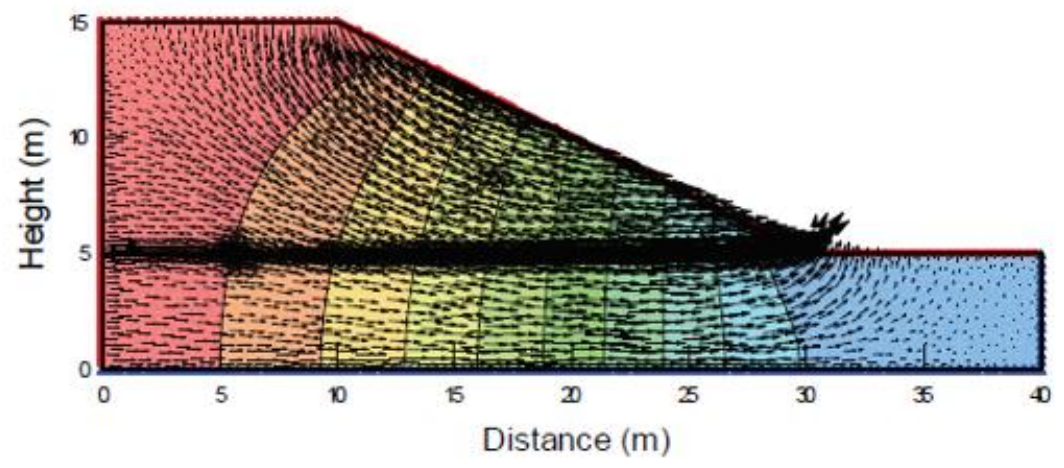

Fig.3.4 Flow field and equipotent lines from SEEP/W

Similar pore pressure conditions were also studied in PLAXIS. The use of hydrostatic pore pressure distribution resulted in a lower FOS compared to steady-sate groundwater calculation.

The resulted CSS and FOS are depicted in Fig. 3.5 and Fig. 3.6 for pore pressure from hydrostatic distributions and seepage analyses, respectively.

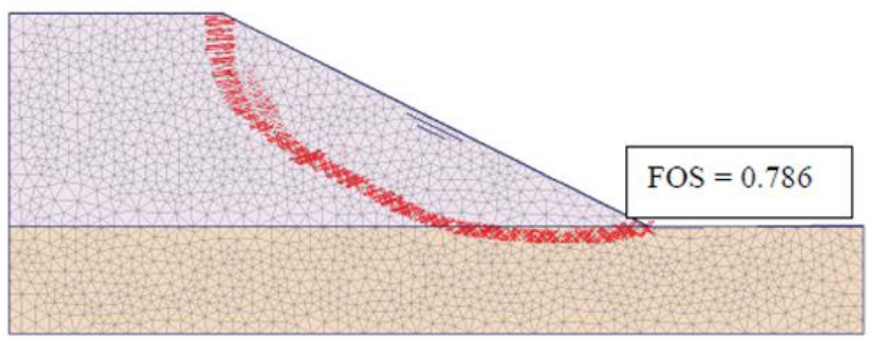

Fig.3.5 CSS and FOS computed in PLAXIS for Case 2a (hydrostatic pore pressure)

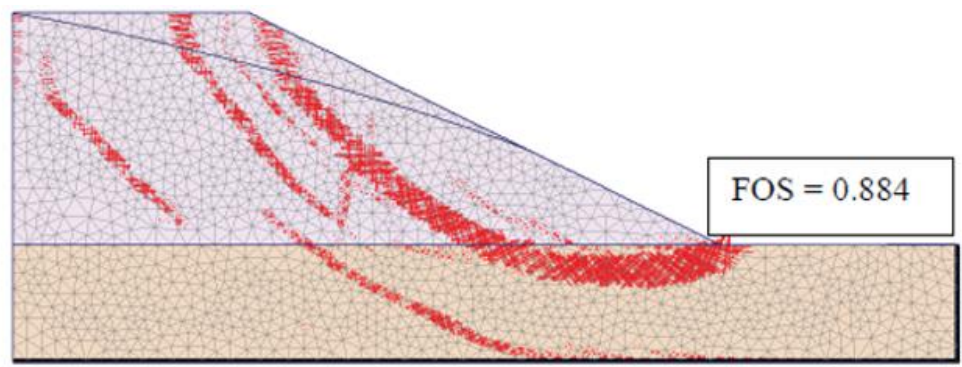

Fig.3.6 CSS and FOS computed in PLAXIS for Case 2b (seepage analysis)

\section{Conclusions}


Today, the two approaches of stability analyses, one based on limit equilibrium (LE) formulations and the other based on finite element (FE) principles are widely used in practice.

The basic physics of stress-strain relationship, which is lacking in LE methods, has been well covered by the FE methods. As a result, complicated geotechnical computations can easily be performed. In addition, FE analysis can simulate stress concentrated problems and deformation compatibility, which have been experienced problematic in LE analysis. This has been one of the advantages of FE calculations. On the other hand, LE methods have been applied for many years. Hence, they are wellestablished and common in practice. User-friendliness, simplicity and relatively good FOS for a particular case are the advantages of the LE methods.

The scope of this study was to compare various stability evaluation methods. Accordingly, most common LE approaches were compared with the advanced LE (MP) method. Similarly, the differences in FOS computed from LE and FE analyses were compared based on a simple slope considering various load cases. In addition, two real slopes in a case study were analyzed for the recorded minimum-maximum GWT, pseudo-static and dynamic conditions. Moreover, the stability evaluations of these slopes were based on both LE (M-P) and FE (PLAXIS) calculation approaches, which both utilized shear strength parameters from advanced triaxial tests.

Similarly, Mohr-Coulomb model was applied in both approaches. The following conclusions are hence derived based on the reported work on both idealized and real slopes

\section{References}

[1] Altea \& Geostudio 2000 Shpk 2011 Report on the geological and geotechnical conditions of "Vlora by-pass"

[2] Aryal, K. Sandven, R. and Nordal, S. (2005). Limit Equilibrium and Finite Element Methods.

[3] Aryal, K., Rohde, J. K. and Sandven, R. (2004). Slope Stability, Stabilization and Monitoring

[4] Athanasiu, C. (2005). Discussions on influencing factors on shear strength parameters.

[5] Bishop, A. W. (1955). The use of slip circles in stability analysis of slopes.

[6] Fredlund, D. G. and Krahn, J. (1977). Comparison of slope stability methods of analysis.

[7] Janbu, N. (2001). Slope Stability Procedures used in Landslide Evaluations in Practice

[8] Janbu, N. (1954a). Stability analysis of Slopes with Dimensionless Parameters.

[9] Janbu, N. (1954b). Application of Composite Slip Surface for Stability Analysis.

[10] Krahn, J. (2004). Stability Modelling with SLOPE/W. An Engineering Methodology

[11] Krahn, J. (2003). The limits of limit equilibrium analysis. 
[12] Leshchinsky, D., and Baker, R. (1986). Three dimensional slope stability

[13] Leshchinsky, D., and Huang, C. (1992). Generalised three dimensional slope stability analyses.

[14] Morgenstern, N. R. and Price, V. E. (1965). The Analysis of the Stability of General Slip Surfaces.

[15] Nash, D. (1987). Comprehensive Review of Limit Equilibrium Methods of Stability Analysis.

[16] PLAXIS (2004). Finite Element Code for Soil and Rock Analyses.

[17] SLEEP/W (2005). Seepage analyses.

[18] SLIDE (2003). Stability analysis for soil and rock slopes.

[19] SLOPE/W (2002). Stability Analysis.

[20] Spencer, E. (1967). A method of Analysis of the Stability 